\title{
Quantization Ambiguity and Supersymmetric Ground State Wave Functions
}

\author{
Kazunori Takenaga * \\ Niels Bohr Institute, Copenhagen University, \\ Blegdamsvej 17, DK-2100 Copenhagen $\emptyset$ Denmark
}

\begin{abstract}
Supersymmetric ground state wave functions of a model of supersymmetric quantum mechanics on $S^{1}$ (supersymmetric simple pendulum) are studied. Supersymmetry can be broken due to the existence of an undetermined parameter, which is interpreted as a gauge field and appears as a firm consequence of quantization on a space with a nontrivial topology such as $S^{1}$. The breaking does not depend on the leading term of the superpotential, contrary to the usual case. The mechanism of supersymmetry breaking is similar to that through boundary conditions of fields in supersymmetric quantum field theory on compactified space. The supersymmetric harmonic oscillator is realized in the limit of the infinite radius of $S^{1}$ with the strength of the oscillator being constant.
\end{abstract}

NBI-HE-00-16

March 2000

*email: takenaga@alf.nbi.dk 


\section{Introduction}

In this paper we study how the quantization ambiguity, which implies that quantization on space with a nontrivial topology such as $S^{1}$ inevitably yields an undetermined parameter into the theory [1], affects the supersymmetric ground state wave functions of a model of supersymmetric quantum mechanics on $S^{1}$.

Quantum mechanics on $S^{1}$ was studied for the first time in the path-integral formalism [2][3]. We observe that the aforementioned parameter appears as a phase factor in the Feynman kernel due to the nontrivial topology of configuration space and, as a result, there are many distinct propagators labeled by the parameter. One can also consider the same effect in the Lagrangian by adding a total derivative term whose coefficient is given by the parameter. Accordingly, the canonical momentum is shifted by the amount of the parameter, so that it can be interpreted as a constant gauge field. The total derivative term has physical implications at the quantum level for space with nontrivial topology [4.

Quantization on $S^{1}$ is much different from that on one-dimensional Euclidean space. In the language of canonical formalism, the latter case is that the representation of the canonical algebra is uniquely determined up to a unitary equivalent representation. There is essentially one quantum mechanics on the space. In the former case, however, there is an infinite number of inequivalent representations of the fundamental algebra, which is introduced as a generalization of the canonical algebra by Ohnuki and Kitakado [5] in order to formulate quantum mechanics on $S^{1}$. As a result, there exists various quantum mechanics on $S^{1}$.

The various quantum mechanics on $S^{1}$ are parametrized by the undetermined parameter. The parameter is interpreted as a constant gauge field [5] [6]. The gauge field can exist and has effects on observables at the quantum level. It is the existence of the gauge field that leads to the various quantum mechanics on $S^{1}$. It may be interesting to study the possible effects of the gauge field on the supersymmetric ground state (zero-energy state) wave functions of supersymmetric quantum mechanics on $S^{1}$. The zero-energy state wave functions can be obtained in closed form [7] because, thanks to the supersymmetry algebra satisfied by the system, the wave functions are obtained by solving simple first-order equations in many cases. Therefore, it may be possible to study the effects as analytically as possible.

Supersymmetric quantum mechanics has been studied in great detail and applied to many physics fields [8]. Actually, it provides us with an example of the dynamical supersymmetry breaking by instantons in certain models [9] 10 11 [12. In those models, the normalizability of the supersymmetric ground state wave function crucially depends on the leading term in the superpotential, by which we determine whether or not the supersymmetry is broken. The semiclassical instanton approximation has been used to 
estimate the ground state energy for the system with broken supersymmetry円.

In this paper we will find an another mechanism of supersymmetry breaking. The very existence of the gauge field twists boundary conditions of supersymmetric ground state wave functions. For certain values of the gauge field, the wave functions do not satisfy a required periodic boundary condition and become unphysical though they are normalizable. The supersymmetry breaking does not depend on the structure of the superpotential, unlike the usual supersymmetry breaking discussed in supersymmetric quantum mechanics. Supersymmetry can be broken by the gauge field, that is, the quantization ambiguity.

In the next section we shall introduce a model of supersymmetric quantum mechanics on $S^{1}$ after reviewing briefly the quantum mechanics on $S^{1}$ formulated by Ohnuki and Kitakado. And then, we shall discuss how the gauge field affects the supersymmetric ground state wave functions and how it yields supersymmetry breaking. We shall also study an infinite limit of the radius of $S^{1}$. The supersymmetric harmonic oscillator is realized in the limit with the strength of the oscillator being constant. The final section is devoted to conclusions and a discussion, where we shall also discuss the similarities between our mechanism of supersymmetry breaking and that through the boundary conditions of fields in supersymmetric quantum field theory on compactified space.

\section{Supersymmetric Quantum Mechanics on $S^{1}$}

We shall study effects of the quantization ambiguity, which implies that quantization on a space with nontrivial topology yields an undetermined parameter, on supersymmetric ground state wave functions of supersymmetric quantum mechanics on $S^{1}$. Let us consider a system in which there is the fermionic operator $\hat{Q}_{i}$ that commutes with the Hamiltonian $\hat{H}$ and satisfies the supersymmetry algebra

$$
\left[\hat{Q}_{i}, \hat{H}\right]=0, \quad\left\{\hat{Q}_{i}, \hat{Q}_{j}\right\}=\delta_{i j} \hat{H}, \quad i=1, \cdots N
$$

$N=2$ is the simplest case and it is of our interest.

Since the Hamiltonian is positive semidefinite, a supersymmetric state $\hat{Q}_{i}|\Psi\rangle=0$ is automatically a zero-energy ground state. Conversely, if we have a zero-energy state, it has to be a supersymmetric ground state. Thanks to this property, finding supersymmetric ground states is reduced to solving simple first-order equations instead of solving the second-order equation $\hat{H}|\Psi\rangle=0$. A key point for our study is that the fermionic operator $\hat{Q}_{i}(i=1,2)$ should be written in terms of the operators which are appropriate to describe the quantum mechanics on $S^{1}$ as shown in the subsection 2.2. And we shall study the supersymmetric ground state wave functions of such a system 2 .

\footnotetext{
${ }^{1}$ Estimating the ground state energy is a subtle problem. Actually, it is reported in 13 [14 that the instanton calculation is very limited though it gives us an excellent estimation in some cases and the breakdown of symmetry is caused by the interplay of perturbative and nonperturbative effects.

${ }^{2}$ Concerning supersymmetric quantum mechanics and the ground state wave functions, it is known
} 


\subsection{Quantum Mechanics on $S^{1}$}

Before we proceed to a model of supersymmetric quantum mechanics on $S^{1}$, it may be important and instructive to review briefly the Ohnuki-Kitakado formulation of quantum mechanics on $S^{1}$ [5]. Those who are familiar with their formulation can skip this subsection and go directly to the subsection 2.2 where the supersymmetric quantum mechanics on $S^{1}$ is introduced. The discussions below are based on a paper [6] in a part of which the quantum mechanics on $S^{1}$ is summarized clearly.

The quantum mechanics on $S^{1}$ is defined by a self-adjoint operator $\hat{G}$ and a unitary operator $\hat{W}$ satisfying the commutation relation

$$
[\hat{G}, \hat{W}]=\hbar \hat{W}
$$

The operators $\hat{G}, \hat{W}$, and $\hat{W}^{\dagger}$ generate an algebra. Let us construct its representation. We shall start with an eigenvalue equation

$$
\hat{G}|\alpha\rangle=\hbar \alpha|\alpha\rangle \quad \text { with } \quad\langle\alpha \mid \alpha\rangle=1,
$$

where an eigenvalue $\alpha$ is a real number. It is easy to see that $\hat{W}\left(\hat{W}^{\dagger}\right)$ raises (lowers) the eigenvalues of $\hat{G}$

$$
\hat{G} \hat{W}|\alpha\rangle=\hbar(\alpha+1) \hat{W}|\alpha\rangle, \quad \hat{G} \hat{W}^{\dagger}|\alpha\rangle=\hbar(\alpha-1) \hat{W}^{\dagger}|\alpha\rangle
$$

A state vector defined by

$$
|n+\alpha\rangle \equiv \hat{W}^{n}|\alpha\rangle, \quad n=\text { integer }
$$

is also an eigenstate of $\hat{G}$ :

$$
\hat{G}|n+\alpha\rangle=\hbar(n+\alpha)|n+\alpha\rangle \text {. }
$$

For fixed $\alpha$, our Hilbert space, denoted by $\mathcal{H}_{\alpha}$ where the two operators $\hat{G}, \hat{W}$ are defined, is given by completing the vector space of linear combinations of $|n+\alpha\rangle(n=0, \pm 1, \pm 2, \cdots)$. The set of state vectors forms the orthocomplete system in $\mathcal{H}_{\alpha}$. Therefore, we have

$$
\langle m+\alpha \mid n+\alpha\rangle=\delta_{m n}, \quad \sum_{n=-\infty}^{+\infty}|n+\alpha\rangle\langle n+\alpha|=\mathbf{1}_{\alpha}
$$

where $\mathbf{1}_{\alpha}$ is an identity operator in $\mathcal{H}_{\alpha}$. Equation (6) and $\hat{W}|n+\alpha\rangle=|n+1+\alpha\rangle$ define an irreducible representation of the algebra (2) on $\mathcal{H}_{\alpha}$. The classification of the irreducible representation of the algebra may be done by noting that (i) $\mathcal{H}_{\alpha}$ and $\mathcal{H}_{\beta}$ are

that a supersymmetric quantum mechanical system can be constructed by using the ground state wave functions of a nonsupersymmetric Hamiltonian 15]. In this approach the superpotential can be given by the ground state wave function of the system. And the approach is extended to generally covariant systems such as relativistic particles interacting with external gauge fields and gravitational fields etc. [16. 
unitary equivalent Hilbert space if and only if $\alpha-\beta=$ integer and (ii) for an arbitrary irreducible representation $\mathcal{H}$ of the algebra, there exists a real number $\alpha$ such that $\mathcal{H}$ is the unitary equivalent of $\mathcal{H}_{\alpha}$. Thus, the classification is completed; that is, all the inequivalent irreducible representations are given by the Hilbert space $\mathcal{H}_{\alpha}(0 \leq \alpha<1)$. It should be emphasized that the algebra (2) has an infinite number of inequivalent representations characterized by an undetermined parameter $\alpha$, as contrary to the usual irreducible representation of the canonical algebra on one-dimensional Euclidean space.

So far, we have constructed the $\hat{G}$-diagonal representation. One can also go to the $\hat{W}$ diagonal representation by which we will obtain wave functions in the quantum mechanics on $S^{1}$. For fixed representation space $\mathcal{H}_{\alpha}$, since $\hat{W}$ is a unitary operator, the eigenvalue equation for it may be written as

$$
\hat{W}|\theta\rangle=\mathrm{e}^{i \theta}|\theta\rangle
$$

Its solution is

$$
|\theta\rangle=\kappa(\theta) \sum_{n=-\infty}^{+\infty} \mathrm{e}^{-i n \theta}|n+\alpha\rangle,
$$

where $\theta$ is a real parameter and $\kappa(\theta)$ is an arbitrary complex-valued function satisfying $|\kappa(\theta)|=1$ and $\kappa(\theta+2 \pi)=\kappa(\theta)$. It is not difficult to show that

$$
\begin{aligned}
|\theta+2 \pi n\rangle & =|\theta\rangle, \quad n=\text { integer, } \\
\left\langle\theta \mid \theta^{\prime}\right\rangle & =2 \pi \sum_{n=-\infty}^{n=+\infty} \delta\left(\theta-\theta^{\prime}+2 \pi n\right), \\
\int_{0}^{2 \pi} \frac{d \theta}{2 \pi}|\theta\rangle\langle\theta| & =\sum_{n=-\infty}^{+\infty}|n+\alpha\rangle\langle n+\alpha|=\mathbf{1}_{\alpha}, \\
\exp \left(-i \lambda \frac{\hat{G}}{\hbar}\right)|\theta\rangle & =\mathrm{e}^{-i \lambda \alpha} \kappa(\theta) \kappa^{*}(\theta+\lambda)|\theta+\lambda\rangle,
\end{aligned}
$$

where $\mathbf{1}_{\alpha}$ is an identity operator in $\mathcal{H}_{\alpha}$. These correspond to periodicity, orthonormality, completeness, and translation for the eigenstate of $\hat{W}$. Let us note that it may be possible from Eqs. (8) and (13) to identify $\hat{G}$ and $\hat{W}$ with the momentum and the position operators on $S^{1}$, respectively.

Now, let $|\psi\rangle$ be a state vector and we define a wave function $\psi(\theta)$ on $S^{1}$ as follows

$$
\psi(\theta) \equiv\langle\theta \mid \psi\rangle
$$

Taking the inner product of Eq. (13) with $|\psi\rangle$, we obtain

$$
\left\langle\theta\left|\exp \left(i \lambda \frac{\hat{G}}{\hbar}\right)\right| \psi\right\rangle=\mathrm{e}^{i \lambda \alpha} \kappa^{*}(\theta) \kappa(\theta+\lambda)\langle\theta+\lambda \mid \psi\rangle
$$

from which the $\hat{W}$-diagonal representation of $\hat{G}$ is given by

$$
\langle\theta|\hat{G}| \psi\rangle=\left[-i \hbar \frac{\partial}{\partial \theta}-i \hbar \kappa^{*}(\theta) \frac{\partial \kappa(\theta)}{\partial \theta}+\hbar \alpha\right] \psi(\theta)
$$


We also obtain, from Eq. (8),

$$
\langle\theta|\hat{W}| \psi\rangle=\mathrm{e}^{i \theta} \psi(\theta) .
$$

The inner product on $S^{1}$ is expressed in terms of the wave function as

$$
\langle\chi \mid \psi\rangle=\int_{0}^{2 \pi} \frac{d \theta}{2 \pi} \chi^{*}(\theta) \psi(\theta) .
$$

Thus, the representation of Hilbert space, which is defined by Eqs. (16) and (17), is the space of the square integrable function on $S^{1}$. Let us note that all wave functions have to satisfy the periodic boundary condition $\psi(\theta+2 \pi n)=\psi(\theta)$, which is a direct consequence of Eq. (10). This periodicity is essential when we study the supersymmetric ground state wave functions of the supersymmetric quantum mechanics on $S^{1}$.

Let us next present the physical meaning of the parameter $\alpha$. To this end, let us redefine $\kappa(\theta)$ by utilizing the arbitrariness of it in such a way that $\kappa(\theta)=\omega(\theta) \kappa^{\prime}(\theta)$, where $\omega(\theta)$ has to satisfy $|\omega(\theta)|=1$ and $\omega(\theta+2 \pi)=\omega(\theta)$. It follows that $|\theta\rangle=\omega(\theta)|\theta\rangle^{\prime}$, so that the transformed wave function $\psi^{\prime}(\theta)$ is given by

$$
\psi^{\prime}(\theta)=\omega(\theta) \psi(\theta) .
$$

According to this redefinition, the $\hat{W}$-diagonal representation for $\hat{G}$ becomes

$$
{ }^{\prime}\langle\theta|\hat{G}| \psi\rangle=\left[-i \hbar \frac{\partial}{\partial \theta}+A^{\prime}(\theta)\right] \psi(\theta),
$$

where we have defined

$$
A^{\prime}(\theta) \equiv A(\theta)+i \hbar \omega^{*}(\theta) \frac{\partial \omega(\theta)}{\partial \theta}, \quad A(\theta) \equiv-i \hbar \kappa^{*}(\theta) \frac{\partial \kappa(\theta)}{\partial \theta}+\hbar \alpha .
$$

Equations (19) and (21) stand for the gauge transformation. Therefore, the parameter $\alpha$ has the meaning of the gauge field. It is easy to see that the gauge field has the properties (i) $A(\theta)$, assumed to be an arbitrary real-valued function satisfying the periodic boundary condition $A(\theta+2 \pi)=A(\theta)$, can always be made a constant function $A^{\prime}(\theta)=\alpha$ by a gauge transformation and (ii) for two constant functions $A^{\prime}(\theta)=\alpha$ and $A^{\prime}(\theta)=\beta$, these are connected by a unique gauge transformation if and only if $\beta-\alpha$ is an integer. Thus, we arrive at an important conclusion that all the inequivalent gauge fields are given by $A_{\alpha} \equiv \alpha(0 \leq \alpha<1)$. Hereafter, we choose $\kappa(\theta)=1$ for simplicity.

It is a very special feature of the quantum mechanics on $S^{1}$ that the inequivalent gauge field is restricted to be $0 \leq \alpha<1$. Another way of looking at it is that if we perform a gauge transformation by $\psi(\theta) \rightarrow \psi^{\prime}(\theta)=\mathrm{e}^{i n \theta} \psi(\theta)$, we see that the gauge fields $A(\theta)$ and $A(\theta)-n \hbar$ are equivalent for $n=$ integer. $n$ has to be restricted to be an integer;

\footnotetext{
${ }^{3}$ Dirac's approach to the quantization for a constrained system does not yield an infinite number of inequivalent representations; that is, it corresponds to only $\alpha=0$. Quantization and embedding $S^{1}$ into higher dimensional space $\mathbf{R}^{2}$ is not a "commutative" procedure relating to each other. Let us also note that the Ohnuki-Kitakado formulation is independent of the dynamics, in contrast to Dirac's approach.
} 
otherwise, the transformed wave function $\psi^{\prime}(\theta)$ does not satisfy the required periodic boundary condition. Therefore, the inequivalent gauge field is given by $0 \leq A(\theta)<\hbar$, which means $0 \leq \alpha<1$. Let us note that the gauge transformation by $\mathrm{e}^{\text {in } \theta}$ with $n=$ noninteger is a singular gauge transformation and is strictly forbidden.

Different values of the gauge field give different quantum mechanics on $S^{1}$. It may be helpful to note that the gauge field $\alpha$ may correspond to the magnitude of the magnetic flux $e \Phi / 2 \pi \hbar c$ through $S^{1}$ in the Aharanov-Bohm effect. The different magnitude of the flux actually gives different physics.

\subsection{Supersymmetric Simple Pendulum}

Now, we are ready to introduce a model of the supersymmetric quantum mechanics on $S^{1}$. According the discussion above, the two operators $\hat{G}$ and $\hat{W}$, which correspond to the momentum and the position of a particle on $S^{1}$, are fundamental. It may be natural to construct a quantum Hamiltonian in terms of these operators. The Hamiltonian in our model is assumed to satisfy the supersymemtry algebra (1), so that the fermionic operators $\hat{Q}_{i}(i=1,2)$ also have to be given in terms of them. We will discuss the classical counterpart of the quantum hamiltonian constructed in this way later.

Let us define the fermionic operator $\hat{Q}_{i}(i=1,2)$ by

$$
\begin{aligned}
\hat{Q} & \equiv \frac{1}{\sqrt{2}}\left(\hat{Q}_{1}+i \hat{Q}_{2}\right) \\
& =\left(\frac{1}{\sqrt{2 m} R} \hat{G}+i V\left(\hat{W}, \hat{W}^{\dagger}\right)\right) \hat{\xi} \equiv \hat{q} \hat{\xi} \\
\hat{\bar{Q}} & \equiv \frac{1}{\sqrt{2}}\left(\hat{Q}_{1}-i \hat{Q}_{2}\right) \\
& =\left(\frac{1}{\sqrt{2 m} R} \hat{G}-i V\left(\hat{W}, \hat{W}^{\dagger}\right)\right) \hat{\bar{\xi}} \equiv \hat{q}^{\dagger} \hat{\bar{\xi}}
\end{aligned}
$$

$V\left(\hat{W}, \hat{W}^{\dagger}\right)$, which is called the superpotential hereafter, is a hermitian operator in terms of $\hat{W}$ and $\hat{W}^{\dagger}$. Here $m$ and $R$ stand for the mass of a particle and the radius of $S^{1}$, respectively. The fermionic variables $\hat{\xi}, \hat{\bar{\xi}}$ satisfy the algebra

$$
\{\hat{\xi}, \hat{\bar{\xi}}\}=1, \quad \hat{\xi}^{2}=\hat{\bar{\xi}}=0 .
$$

Then, the Hamiltonian is given by

$$
\begin{aligned}
\hat{H} & =\{\hat{Q}, \hat{\bar{Q}}\} \\
& =\frac{1}{2 m R^{2}} \hat{G}^{2}+V^{2}\left(\hat{W}, \hat{W}^{\dagger}\right) \\
& -\frac{i}{\sqrt{2 m} R}\left(\hat{G} V\left(\hat{W}, \hat{W}^{\dagger}\right)-V\left(\hat{W}, \hat{W}^{\dagger}\right) \hat{G}\right)[\hat{\xi}, \hat{\bar{\xi}}]
\end{aligned}
$$

where we have used Eq. (24). 
Here, it may be necessary to discuss the classical counterpart of the quantum Hamiltonian (25). To this end, let us note that the fundamental algebra (2) may be actually inferred by the classical Poisson's brackets for the angle variable $\theta$ and the correponding momentum $P_{\theta}$ in the polar coordinate:

$$
\left\{P_{\theta}, \mathrm{e}^{i \theta}\right\}_{P}=-i \mathrm{e}^{i \theta} .
$$

If we replace the classical Poisson's brackets by the commutation relation divided by $i \hbar$, we obtain the fundamental algebra (2) by identifying $\mathrm{e}^{i \theta}$ and $P_{\theta}$ with $\hat{W}$ and $\hat{G}$, respectively. This is the same identification stated earlier. Therefore, in the classical limit we may replace $\hat{W}$ by e $\mathrm{e}^{i \theta}$ and $\hat{G}$ by $P_{\theta}$. According to these replacements, we obtain a classical Hamiltonian, ignoring the fermionic variables $\hat{\xi}, \hat{\bar{\xi}}$ :

$$
\hat{H} \rightarrow H_{c l}=\frac{P_{\theta}^{2}}{2 m R^{2}}+V^{2}\left(\mathrm{e}^{i \theta}, \mathrm{e}^{-i \theta}\right) .
$$

If we choose $V\left(\mathrm{e}^{i \theta}, \mathrm{e}^{-i \theta}\right)$ as

$$
V\left(\mathrm{e}^{i \theta}, \mathrm{e}^{-i \theta}\right)=\sqrt{\frac{m g_{N} R}{2}} \sin \theta,
$$

the classical Hamiltonian describes a simple pendulum with angle $2 \theta$. Here $g_{N}$ is the gravitation accelerator constant.

On the other hand, given the superpotential (28), the quantum counterpart of it is obtained by

$$
V\left(\hat{W}, \hat{W}^{\dagger}\right)=\sqrt{\frac{m g_{N} R}{2}}\left(\frac{\hat{W}-\hat{W}^{\dagger}}{2 i}\right) .
$$

Having this superpotential, the model of the supersymmetric quantum mechanics on $S^{1}$, that is, the supersymmetric simple pendulum, is given by the Hamiltonian

$$
\hat{H}=\frac{1}{2 m R^{2}} \hat{G}^{2}+\frac{m g_{N} R}{2} \sin ^{2} \theta-\frac{\hbar}{2} \sqrt{\frac{g_{N}}{R}} \cos \theta[\hat{\xi}, \hat{\bar{\xi}}]
$$

in the $\hat{W}$-diagonal representation. It is understood that $\hat{G}=-i \hbar \partial / \partial \theta+\hbar \alpha$ and $\alpha$ is the gauge field discussed in the previous subsection.

Let us study the supersymmetric ground state wave functions of the supersymmetric simple pendulum whose hamiltonian is given by Eq. (30). It follows from the algebra (1) that the supersymmetric ground states must be zero-energy states satisfied by

$$
\hat{Q}|\Psi\rangle=0 \quad \text { and } \quad \hat{\bar{Q}}|\Psi\rangle=0 .
$$

Let us introduce a matrix representation for the fermionic variables. It is easy to see that the matrix representations given by

$$
\hat{\xi}=\left(\begin{array}{cc}
0 & 0 \\
1 & 0
\end{array}\right), \quad \hat{\bar{\xi}}=\left(\begin{array}{cc}
0 & 1 \\
0 & 0
\end{array}\right)
$$


satisfy the algebra (24). Then, it follows that

$$
[\hat{\xi}, \hat{\bar{\xi}}]=-\left(\begin{array}{cc}
1 & 0 \\
0 & -1
\end{array}\right) \equiv-\sigma^{3}
$$

Using these, the Hamiltonian (30) becomes

$$
\begin{aligned}
\hat{H} & =\left(\frac{1}{2 m R^{2}} \hat{G}^{2}+\frac{m g_{N} R}{2} \sin ^{2} \theta\right) \mathbf{1}_{2 \times 2}+\frac{\hbar}{2} \sqrt{\frac{g_{N}}{R}} \sigma^{3} \cos \theta \\
& =\left(\begin{array}{cc}
\hat{q}^{\dagger} \hat{q} & 0 \\
0 & \hat{q} \hat{q}^{\dagger}
\end{array}\right) \equiv\left(\begin{array}{cc}
\hat{H}_{+} & 0 \\
0 & \hat{H}_{-}
\end{array}\right) .
\end{aligned}
$$

In the matrix representation, the hamiltonian is a $2 \times 2$ matrix.

Since the Hamiltonian (33) commutes with an operator $\hat{S}^{F} \equiv \sigma^{3} / 2$, the eigenstates of the Hamiltonian is labeled by the eigenvalues of $\hat{S}^{F}$. Let us call the two states $|+\rangle$ and $|-\rangle$ fermion numbers $+1 / 2$ and $-1 / 2$, respectively $]$. The state vector is, now, a two-component vector

$$
|\Psi\rangle=\left(\begin{array}{l}
|+\rangle \\
|-\rangle
\end{array}\right)
$$

In the $\hat{W}$-diagonal representation, it may be written as

$$
\Psi(\theta)=\left(\begin{array}{c}
\psi_{+\frac{1}{2}}(\theta) \\
\psi_{-\frac{1}{2}}(\theta)
\end{array}\right)
$$

The Hamiltonian $\hat{H}$ is diagonalized with respect to the fermion number $\pm 1 / 2$. In this matrix representation, Eq. (31) is read as

$$
\begin{aligned}
& \hat{q} \psi_{+\frac{1}{2}}(\theta)=\left(\frac{1}{\sqrt{2 m} R}\left(-i \hbar \frac{\partial}{\partial \theta}+\hbar \alpha\right)+i \sqrt{\frac{m g_{N} R}{2}} \sin \theta\right) \psi_{+\frac{1}{2}}(\theta)=0, \\
& \hat{q}^{\dagger} \psi_{-\frac{1}{2}}(\theta)=\left(\frac{1}{\sqrt{2 m} R}\left(-i \hbar \frac{\partial}{\partial \theta}+\hbar \alpha\right)-i \sqrt{\frac{m g_{N} R}{2}} \sin \theta\right) \psi_{-\frac{1}{2}}(\theta)=0 .
\end{aligned}
$$

Solutions for Eq. (36) are found to be

$$
\psi_{+\frac{1}{2}}(\theta)=\frac{1}{\sqrt{I_{0}(2 z)}} \exp \left(-i \alpha \theta-\frac{z}{\hbar} \cos \theta\right), \quad \psi_{-\frac{1}{2}}(\theta)=\frac{1}{\sqrt{I_{0}(2 z)}} \exp \left(-i \alpha \theta+\frac{z}{\hbar} \cos \theta\right)
$$

where $I_{0}(2 z)$ in the normalization factor is the zeroth-order modified Bessel function and we have defined a dimensionless parameter

$$
\frac{m R^{2}}{\hbar} \sqrt{\frac{g_{N}}{R}} \equiv \frac{m R^{2}}{\hbar} \omega \equiv \frac{z}{\hbar}
$$

These are normalizable solutions. Thus, one may say that the zero-energy states, that is, supersymmetric ground states, exist in the model and the supersymmetry is unbroken.

\footnotetext{
${ }^{4}$ One may call the two states spin-up and spin-down states. Or by introducing $\hat{f}=\frac{1}{2}+\frac{1}{2}[\hat{\xi}, \hat{\bar{\xi}}]$ whose eigenvalues are 0,1 , one may denote them by fermion numbers 0,1 .
} 
This is, however, a hasty conclusion. In addition to the normalizability, all the wave functions have to satisfy the periodic boundary condition $\Psi(\theta+2 \pi)=\Psi(\theta)$, that is, $\psi_{ \pm \frac{1}{2}}(\theta+2 \pi)=\psi_{ \pm \frac{1}{2}}(\theta)$, which follows from Eq. (10) ๒. It is easy to see from Eq. (37) that

$$
\psi_{ \pm \frac{1}{2}}(\theta+2 \pi)=\mathrm{e}^{-i 2 \pi \alpha} \psi_{ \pm \frac{1}{2}}(\theta)
$$

The boundary condition for the zero-energy state wave functions is twisted by the gauge field $\alpha$. The zero-energy state wave functions do not satisfy the required periodic boundary condition except for $\alpha=$ integer. Since the inequivalent representation is given by $0 \leq$ $\alpha<1$, they are inconsistent with the periodic boundary condition and become unphysical wave functions for $0<\alpha<1$. Therefore, the supersymmetry can be broken due to the gauge field $\alpha$. Let us note that the Witten index $\operatorname{Tr}(-1)^{\hat{f}}=n_{B}^{E=0}-n_{F}^{E=0}$ vanishes in our model. It is easy to see that $n_{B}^{E=0}=n_{F}^{E=0}=1$ for $\alpha=$ integer and that $n_{B}^{E=0}=n_{F}^{E=0}=0$ for $\alpha=$ noninteger.

Unlike the usual supersymmetry breaking, in which the leading term of the superpotential determines whether or not supersymmetry is broken, our breaking of supersymmetry does not depend on the structure of the superpotential. It is entirely due to the existence of the gauge field $\alpha$, which is an inevitable consequence of the quantization ambiguity when one quantizes the theory on topologically nontrivial space such as $S^{1}$. The gauge field has the effect of twisting the boundary conditions of the zero-energy state wave functions. Among the various supersymmetric quantum mechanics on $S^{1}$ led by the gauge field, it includes theories with broken supersymmetry due to the gauge field. Let us note that in this context there is no mechanism to determine the values of $\alpha$ or what values of $\alpha$ we should take.

Note that for noninteger values of the gauge field $\alpha$, the gauge field can not be removed in Eq. (36) by the gauge transformation

$$
\psi_{ \pm \frac{1}{2}}(\theta) \longrightarrow \psi_{ \pm \frac{1}{2}}^{\prime}(\theta)=\mathrm{e}^{-i \alpha \theta} \psi_{ \pm \frac{1}{2}}(\theta)
$$

As we stated before, the inequivalent gauge field is given by $0 \leq \alpha<1$. Any gauge field in this range cannot be connected by a regular gauge transformation with $\mathrm{e}^{i n \theta}(n=$ integer). Only a singular gauge transformation can do it, but it destroys the required periodic boundary condition for the transformed wave function. The singular gauge transformation is strictly forbidden, so that the gauge field cannot be gauged away.

Let us briefly comment on the ground state energy. We have a physical supersymmetric ground state wave function for $\alpha=$ integer, so that the ground state energy is exactly zero. On the other hand, for $0<\alpha<1$, supersymmetry is broken. The ground state energy is nonzero (positive). Estimating the ground state energy is a subtle problem as studied in 13 14.

\footnotetext{
${ }^{5}$ Let us note that the two-component spinor state (34) does not have a minus sign under $2 \pi$ rotation in this case because the rotation is done by the usual rotation matrix in two dimensions.
} 
The Hamiltonian (33) cannot be solved analytically. The bosonic potential $\frac{m g_{N} R}{2} \sin ^{2} \theta$ is periodic and the classical vacuum has a periodic structure. One may expect that there is a instantonlike classical solution, which gives a finite Euclidean action, connecting the two vacua with different fermion numbers $( \pm 1 / 2)$. Actually, there exists such a classical solution in our model. It is given by $\cos \theta_{c l}(\tau)= \pm \tanh \left(\omega\left(\tau-\tau_{0}\right)\right)$ with the classical Euclidean action being $-2 z / \hbar$. And the fermion zero mode exists in this classical background. Therefore, we expect tunneling to occur between the two vacua. According to the semiclassical argument, the tunneling effect shifts the ground state energy to give an exponentially small amount of energy in the form of $\exp (-2 z / \hbar) \times \cos 2 \pi \alpha$ ?

On the other hand, for very small $z$, we can resort to perturbation theory to obtain the energy spectrum of the Hamiltonian. The ground state energy is given by $E_{0} \sim$ $\frac{1}{2 m R^{2}}\left(\alpha^{2}+O\left(z^{2}\right)\right)$, where we have set $\hbar=1$. The gauge field $\alpha$ is a dominant contribution to the ground state energy in this case.

It may be interesting to consider the $R \rightarrow \infty$ limit. So far, we have fixed the radius $R$ of $S^{1}$. If $R$ varies to become large, we expect that the arc of an arbitrary part of $S^{1}$ will approach a straight segment. In the limit of $R \rightarrow \infty$, one-dimensional Euclidean space will be recovered.

In order to study the limit, let us define a variable $x \equiv R \theta$. In terms of this new variable, the Hamiltonian (33) is written as, remembering $\hat{G}=-i \hbar \partial / \partial \theta+\hbar \alpha$ in the $\hat{W}$-diagonal representation,

$$
\hat{H}=\left(\frac{-\hbar^{2}}{2 m}\left(\frac{\partial}{\partial x}+i \frac{\alpha}{R}\right)^{2}+\frac{m g_{N} R}{2} \sin ^{2}\left(\frac{x}{R}\right)\right) \mathbf{1}_{2 \times 2}+\frac{\hbar}{2} \sqrt{\frac{g_{N}}{R}} \sigma^{3} \cos \left(\frac{x}{R}\right) .
$$

If we take the limit of $R \rightarrow \infty$ naively, it becomes trivial for the Hamiltonian to yield the one for a free particle on $S^{1}$ :

$$
\hat{H}=\frac{-\hbar^{2}}{2 m} \frac{\partial^{2}}{\partial x^{2}} \mathbf{1}_{2 \times 2}
$$

In order to obtain an interacting theory, one has to take the limit, keeping a relation given by

$$
\frac{m}{\hbar} \sqrt{\frac{g_{N}}{R}} \equiv \frac{m \omega}{\hbar}=(\text { strength of oscillator })^{2}=\text { const. }
$$

Then, we obtain

$$
\hat{H}=\left(\frac{-\hbar^{2}}{2 m} \frac{\partial^{2}}{\partial x^{2}}+\frac{m \omega^{2}}{2} x^{2}\right) \mathbf{1}_{2 \times 2}+\frac{\hbar \omega}{2} \sigma^{3}+O\left(\frac{1}{R^{2}}\right) .
$$

This is the well-known Hamiltonian for the supersymmetric harmonic oscillator [9] with angular frequency $\omega=\sqrt{g_{N} / R}$. Likewise, by taking the same limit, the fermionic opera-

\footnotetext{
${ }^{6}$ This band structure $\cos 2 \pi \alpha$ can be understood from the effective action obtained by the transition amplitude $K\left(\theta_{f}, t ; \theta_{i}, 0\right)=\left\langle\theta_{f}|\exp (-i \hat{H} t / \hbar)| \theta_{i}\right\rangle=\sum_{n=-\infty}^{+\infty} \int_{n-\text { winding }} \mathcal{D} \theta \exp \left(i S_{\text {eff }} / \hbar\right)$, where the effective action is given by $S_{\text {eff }}=\int d t \frac{m R^{2}}{2}\left(\frac{d \theta}{d t}\right)^{2}-\frac{m g_{N} R}{2} \sin ^{2} \theta+\frac{1}{2} \sqrt{\frac{g_{N}}{R}} \cos \theta\left[\xi, \xi^{*}\right]+i \xi^{*} \frac{d \xi}{d t}-\alpha \frac{d \theta}{d t}$. The "topological" term $\alpha \dot{\theta}$ is the origin of such band structure 17 .
} 
tors $\hat{Q}, \hat{\bar{Q}}$ become

$$
\begin{aligned}
& \hat{Q}=\frac{1}{\sqrt{2 m}}(\hat{p}+i W(\hat{x})) \hat{\xi}+O\left(\frac{1}{R^{2}}\right) \equiv \hat{Q}_{\text {susy }}+O\left(\frac{1}{R^{2}}\right), \\
& \hat{\bar{Q}}=\frac{1}{\sqrt{2 m}}(\hat{p}-i W(\hat{x})) \hat{\bar{\xi}}+O\left(\frac{1}{R^{2}}\right) \equiv \hat{\bar{Q}}_{\text {susy }}+O\left(\frac{1}{R^{2}}\right),
\end{aligned}
$$

where we have defined $W(\hat{x}) \equiv m \omega \hat{x}$ and $\hat{p} \equiv-i \hbar \partial / \partial x$. It is easy to check that the Hamiltonian (43) satisfies the supersymmetry algebra (11) with the supercharges (44) if we use the canonical commutation relation $[\hat{p}, \hat{x}]=-i \hbar$ and Eq. (32). The usual supersymmetry, by which the supersymmetry transformations between boson $(\hat{x})$ and fermion $(\hat{\xi}, \hat{\bar{\xi}})$ are generated, is realized in the limit of $R \rightarrow \infty$ with Eq. (42).

The supersymmetric ground state wave functions for the Hamiltonian (43) are obtained by solving the first-order equation $\hat{Q}_{\text {susy }}|\Psi\rangle=0$ and $\hat{\bar{Q}}_{\text {susy }}|\Psi\rangle=0$. Using the same matrix representation as before, their solutions are

$$
\psi_{+\frac{1}{2}}^{h . o}(x) \sim \exp \left(+\frac{m \omega}{2 \hbar} x^{2}\right), \quad \psi_{-\frac{1}{2}}^{h . o}(x)=\left(\frac{m \omega}{\pi \hbar}\right)^{1 / 4} \exp \left(-\frac{m \omega}{2 \hbar} x^{2}\right) .
$$

There are two candidates for the supersymmetric ground state (zero-energy state) wave functions. The one is physical and its wave function is given by $\psi_{-\frac{1}{2}}^{h . o}(x)$. The other one $\psi_{+\frac{1}{2}}^{h . o}(x)$ is unphysical because of its non-normalizability. Therefore, we have one supersymmetric ground (zero-energy) state. This is consistent with the exact energy spectrum of the Hamiltonian (43). As easily seen from the Hamiltonian, there exists one supersymmetric ground state. In fact, the solutions (45) can be obtained by taking the the limit of $R \rightarrow \infty$ with the relation (42) in Eq. (37). By noting $I_{0}(z) \sim \mathrm{e}^{z} / \sqrt{2 \pi z}$ for large $z$, we obtain

$$
\tilde{\psi}_{+\frac{1}{2}} \sim \exp \left(+\frac{m \omega}{2 \hbar} x^{2}\right), \quad \tilde{\psi}_{-\frac{1}{2}}(x)=\left(\frac{m \omega}{\pi \hbar}\right)^{1 / 4} \exp \left(-\frac{m \omega}{2 \hbar} x^{2}\right)
$$

where we have redefined the normalization as $\tilde{\psi}_{ \pm \frac{1}{2}}(x) d x \equiv \psi_{ \pm \frac{1}{2}}(\theta) \frac{d \theta}{\sqrt{2 \pi R}}$. These are the same as Eqs. (45). The Witten index is $\operatorname{Tr}(-1)^{\hat{f}}=1$ in this case.

A simple generalization of the model is an $N$-component one. The fermionic operators are defined by

$$
\begin{aligned}
& \hat{Q}=\sum_{a=1}^{N}\left(\frac{1}{\sqrt{2 m} R_{a}} \hat{G}_{a}+i \hat{V}_{a}\right) \hat{\xi}_{a} \equiv \sum_{a=1}^{N} \hat{q}_{a} \hat{\xi}_{a} \\
& \hat{\bar{Q}}=\sum_{a=1}^{N}\left(\frac{1}{\sqrt{2 m} R_{a}} \hat{G}_{a}-i \hat{V}_{a}\right) \hat{\bar{\xi}} \equiv \sum_{a=1}^{N} \hat{q}_{a}^{\dagger} \hat{\bar{\xi}}_{a}
\end{aligned}
$$

where $\hat{V}_{a} \equiv \hat{V}_{a}\left(\theta_{1}, \cdots, \theta_{N}\right)$ and $\hat{G}_{a}=-i \hbar \partial / \partial \theta_{a}+\hbar \alpha_{a}$ in the $\hat{W}$-diagonal representation. Let us assume

$$
\left[\hat{G}_{a}, \hat{W}_{b}\right]=\hbar \delta_{a b} \hat{W}_{b}, \quad\left\{\hat{\xi}_{a}, \hat{\bar{\xi}}_{b}\right\}=\delta_{a b}, \quad\left\{\hat{\xi}_{a}, \hat{\xi}_{b}\right\}=0, \quad\left\{\hat{\bar{\xi}}_{a}, \hat{\bar{\xi}}_{b}\right\}=0
$$


Then, the Hamiltonian following from these fermionic operators is

$$
\hat{H}=\sum_{a=1}^{N} \frac{1}{2 m R_{a}^{2}} \hat{G}_{a} \hat{G}_{a}+\hat{V}_{a} \hat{V}_{a}-\sum_{a, b=1}^{N} \frac{i}{\sqrt{2 m} R_{a}}\left[\hat{G}_{a}, \hat{V}_{b}\right]\left[\hat{\xi}_{a}, \hat{\bar{\xi}}_{b}\right] .
$$

The Hamiltonian (50) may describe the supersymmetric quantum mechanics on the torus $T^{N}=S^{1} \otimes \cdots \otimes S^{1}$. As before, $\alpha_{a}(a=1, \cdots, N)$ may be interpreted as the gauge field appearing as a consequence of the quantization on each topological space $S^{1}$.

It is difficult to obtain the exact form of the supersymmetric ground state wave functions of the model. If we, however, restrict ourselves to certain sectors of the model, they can be obtained in closed form such as Eq. (53) [7]. In order to see this, let us define

$$
|-\rangle \equiv|0\rangle, \quad|+\rangle \equiv \prod_{a=1}^{N} \hat{\bar{\xi}}_{a}|0\rangle \quad \text { with } \quad \hat{\xi}_{a}|0\rangle=0 \quad(a=1, \cdots, N) .
$$

Then, $\hat{Q}|-\rangle=\hat{\bar{Q}}|+\rangle=0$ is trivially satisfied, so that in these two sectors, the supersymmetric ground state (zero-energy state) wave functions are obtained in closed form by solving simple first-order equations such as Eqs. (36). Aside from the normalization, the solutions are obtained as

$$
\begin{aligned}
& \Psi^{+}\left(\theta_{1}, \cdots, \theta_{N}\right)=\exp \sum_{a=1}^{N}\left(-i \alpha_{a} \theta_{a}+\frac{\sqrt{2 m} R_{a}}{\hbar} \int^{\theta_{a}} d \bar{\theta}_{a} V_{a}\left(\bar{\theta}_{1}, \cdots, \bar{\theta}_{a}, \cdots, \bar{\theta}_{N}\right)\right)|+\rangle, \\
& \Psi^{-}\left(\theta_{1}, \cdots, \theta_{N}\right)=\exp \sum_{a=1}^{N}\left(-i \alpha_{a} \theta_{a}-\frac{\sqrt{2 m} R_{a}}{\hbar} \int^{\theta_{a}} d \bar{\theta}_{a} V_{a}\left(\bar{\theta}_{1}, \cdots, \bar{\theta}_{a}, \cdots, \bar{\theta}_{N}\right)\right)|-\rangle .(
\end{aligned}
$$

The wave functions have to satisfy the periodic boundary condition $\Psi^{ \pm}\left(\cdots, \theta_{a}+2 \pi, \cdots\right)=$ $\Psi^{ \pm}\left(\cdots, \theta_{a}, \cdots\right)(a=1, \cdots, N)$. If the contributions coming from the superpotential in Eqs. (52) do not spoil the normalizablity and the periodicity of the wave functions, the supersymmetry can be broken for noninteger values of $\alpha_{a}(a=1, \cdots, N)$.

\section{Conclusions and Discussion}

We have applied the Ohknuki-Kitakado formulation of quantum mechanics on $S^{1}$ to the supersymmetric simple pendulum whose Hamiltonian is given by Eq. (33) and satisfies the algebra (11). According to their formulation, an undetermined parameter, which can be interpreted as a constant gauge field, inevitably enters into the theory to yield the various quantum mechanics on $S^{1}$. We have studied the effects of the quantization ambiguity on the supersymmetric ground state wave functions of the model.

We have found that supersymmetry can be broken due to the existence of the gauge field $\alpha$. The gauge field twists the boundary condition of the supersymmetric ground state wave functions. For noninteger values of $\alpha$, they do not satisfy the required periodic boundary condition. As a result, they become unphysical wave functions though they 
are normalizable. The mechanism of supersymmetry breaking is different from the usual supersymmetry breaking discussed in supersymmetric quantum mechanics. The latter depends crucially on the structure, the leading term, of the superpotential, while the former is entirely due to the quantization ambiguity resulting firmly from quantization on a space with nontrivial topology like $S^{1}$.

We have chosen the superpotential $V\left(\hat{W}, \hat{W}^{\dagger}\right)$ in such a way that it becomes a simple pendulum in the classical limit. In principle, one can choose any superpotential as long as it can be written in terms of integer powers of the operators $\hat{W}$ and $\hat{W}^{\dagger}$. Thanks to the factorizable property for finding the supersymmetric ground state wave functions, they are given simply by solving the first order equation (31) and are obtained in closed form

$$
\psi_{ \pm \frac{1}{2}}(\theta)=\exp \left(-i \alpha \theta \mp \frac{\sqrt{2 m} R}{\hbar} \int^{\theta} d \bar{\theta} V\left(\mathrm{e}^{i \bar{\theta}}, \mathrm{e}^{-i \bar{\theta}}\right)\right)
$$

for the general superpotential. Our mechanism of supersymmetry breaking is not altered by the choice of the superpotential if $\exp \left(\int^{\theta} d \bar{\theta} V\left(\mathrm{e}^{i \bar{\theta}}, \mathrm{e}^{-i \bar{\theta}}\right)\right)$ does not violate the periodicity and the normalizability of the wave functions, which is the case for the superpotential satisfying our criterion. Supersymmetry breaking will always occur for noninteger values of $\alpha$. Because of the factorizable property, there is no way to prevent the gauge field from entering into the supersymmetric ground state wave functions and twisting their boundary conditions. The gauge field cannot be removed by a regular gauge transformation.

One may wonder whether all the eigenfunctions of the Hamiltonian (33) become unphysical, that is, those that do not satisfy the periodic boundary condition due to the existence of the gauge field. This is not true. In order to see this, let us consider a free Hamiltonian, ignoring all terms except for $\hat{G}^{2}$. The energy eigenvalue depends on $\alpha$ like $(m+\alpha)^{2}$ and the gauge field produces an effect on the observable at the quantum level [6]. The corresponding eigenfunction satisfying the periodic boundary condition is easily found to be $\mathrm{e}^{i m \theta}$. The ground state wave functions and the other eigenfunctions are obtained by solving essentially different types of differential equations in the system satisfying the supersymmetry algebra (11).

We have also discussed the limit of $R \rightarrow \infty$. One-dimensional Euclidean space is realized. In the limit with the relation (42), we have obtained the supersymmetric harmonic oscillator with angular frequency $\omega=\sqrt{g_{N} / R}$. There exists one physical supersymmetric ground state wave function. The other one, though it is a zero-energy state, is unphysical because of its non-normalizability. These two wave functions are actually obtained by taking the limit in the solutions of the zero-energy wave functions (37). In the limit all the effects of the gauge field $\alpha$ disappear. Then, an infinite number of inequivalent representations is reduced to a unique representation, which is nothing but the representation of the canonical algebra $[\hat{p}, \hat{x}]=-i \hbar$. The fermionic operators become supercharges in the same limit, and they generate supersymmetry transformations between bosons $(\hat{x})$ and fermions $(\hat{\xi}, \hat{\bar{\xi}})$. 
We have also considered the $N$-component generalization of Eq. (25) and studied the supersymmetric ground state wave functions of the model. The Hamiltonian (50) describes the supersymmetric quantum mechanics on the torus $T^{N}$. If we restrict ourselves to the two sectors given by $|-\rangle$ and $|+\rangle$, the wave functions can be obtained in closed form (52) by solving the simple first-order equations. We have found, again, that supersymmetry was able to be broken due to the existence of the gauge field $\alpha_{a} \neq$ integer $(a=$ $1, \cdots, N)$, which appeared as a consequence of the quantization on the each topological space $S^{1}$.

Finally, let us discuss the similarities between our mechanism of supersymmetry breaking and that through boundary conditions of fields for compactified directions in supersymmetric quantum field theory.

Strictly speaking, supersymmetry breaking through boundary conditions is one thing, and that through our mechanism is another. Nevertheless, it may be interesting to discuss the similarities between the two supersymmetry breakings. In the former case, the breaking means that the action is no longer invariant under the supersymmetry transformations. But it does not necessarily mean the nonexistence of the zero-energy state in the system. The supersymmetric harmonic oscillator at finite temperature is one of the examples in which the action is not invariant under the supersymmetry transformations because of the different boundary conditions between the bosons and the fermions; there exists, however, the zero-energy state in the system, which results from $\operatorname{Tr}(-1)^{\hat{f}}=1[18$. In the latter case, we assume that the Hamiltonian satisfies the supersymmetry algebra (更), so that supersymmetry breaking immediately means the nonexistence of physical zeroenergy states in the system, and whether or not supersymmetry is broken is determined definitely by the existence of the zero-energy state.

As seen from Eq. (38), the boundary condition for the $S^{1}$ direction is twisted for $0<\alpha<1$ by $\mathrm{e}^{-i 2 \pi \alpha}$. If we consider a theory at finite temperature, it is equivalent to studying the theory in a space where the Euclidean time direction is compactified on $S^{1}$. It is well known that supersymmetry is broken at finite temperature by the different boundary conditions for the Euclidean time direction between the bosons and the fermions. The boson (fermion) satisfies the (anti)periodic boundary condition. The case of $\alpha=1 / 2$, which actually corresponds to the antiperiodic boundary condition, is similar to the case of supersymmetry breaking at finite temperature.

More generally, if one wishes to break supersymmetry through different boundary conditions between the bosons and the fermions such as the finite temperature case, one can use the boundary condition associated with the $U(1)_{R}$ symmetry [19][20] [21], in which the $U(1)_{R}$ charges are different between the bosons and the fermions in a supermultiplet. If we regard the factor $\mathrm{e}^{-i 2 \pi \alpha}$ in Eq. (38) as a boundary condition that breaks supersymmetry, our mechanism of supersymmetry breaking is quite similar to that through boundary condition associated with the $U(1)_{R}$ symmetry. If one takes this similarity seriously, one 
says that a possible physical origin of supersymmetry breaking through the boundary condition associated with the $U(1)_{R}$ symmetry has been found. Needless to say, in order to confirm this statement, we need to clarify how the quantization ambiguity is realized in (supersymmetric) quantum field theory [22].

\section{Acknowledgments}

I would like to thank Professor Jan Ambj $\phi$ rn for fruitful discussions, encouraging me, and critical comments on this manuscript, and the Niels Bohr Institute for warm hospitality. I would also like to thank Professor Shogo Tanimura (Kyoto University) for valuable discussions on the quantum mechanics on $S^{1}$.

\section{References}

[1] For a pedagogical account on the quantization ambiguity in a path-integral formalism, see, for example, D. McMullan and I. Tsutsui, Ann. Phys. 237 (1995) 269. See also G. W. Mackey, Induced Representations of Group and Quantum Mechanics, Benjamin, New York, 1969.

[2] L. S. Schulman, Phys. Rev. 176 (1968) 1558.

[3] M. G. G. Laidlaw and C. M. DeWitt, Phys. Rev. D3 (1971) 13275.

[4] L. S. Schulman, Techniques and Applications of Path Integration, Wiely, New York, 1981.

[5] Y. Ohnuki and S. Kitakado, J. Math. Phys. 34 (1993) 2827.

[6] S. Tanimura, Prog. Theor. Phys. 90 (1993) 271.

[7] M. Claudson and M. B. Halpern, Nucl. Phys. B250 (1985) 689.

[8] For a review, see, for example, F. Cooper, A. Khare and U. Sukhatme, Phys. Rep. 251 (1995) 267. references therein.

[9] E. Witten, Nucl. Phys. B185 (1981) 513, Nucl. Phys. B202 (1982) 253.

[10] P. Salomonson and J. W. Van Holton, Nucl. Phys. B196 (1982) 509. 
[11] F. Cooper and B. Freedman, Ann. Phys. 146 (1983) 262.

[12] A. Khare and J. Maharana, Zeit. Phys. C23(1984) 191.

[13] R. B. Abbott, Zeit. Phys. C20(1983) 213.

[14] R. B. Abbott and W. J. Zakrzewski, Zeit. Phys. C20(1983) 227.

[15] E. Gozzi, Phys. Lett. B129 (1983) 432.

[16] J. Gamboa and J. Zanelli, Ann. Phys. 188 (1988) 239.

[17] R. Rajaraman, Solitons and Instantons, North-Holland, Amsterdam, 1987.

[18] J. Fuchs, Ann. Phys. 165 (1985) 285.

[19] J. Scherk and J. H. Schwartz, Phys. Lett. B82 (1979) 60.

[20] P. Fayet, Phys. Lett. B159 (1985) 121, Nucl. Phys. B263 (1986) 87.

[21] K. Takenaga, Phys. Lett. B425 (1998) 114, Phys. Rev. D58 (1998) 026004, Erratum, Phys. Rev. D61 (2000) 129902(E).

[22] Work along this direction can be found in Y. S. Wu and A. Zee, Nucl. Phys. B258 (1985) 157, H. Miyazaki and I. Tsutsui, Mod. Phys. Lett. A13(1988) 3017, H. Kobayashi, I. Tsutsui and S. Tanimura, Nucl. Phys. B514 (1998) 667. 